\title{
Review of Control Algorithms for Reconfigurable Battery Systems with an Industrial Example
}

Pinter, Zoltan Mark; Papageorgiou, Dimitrios; Rohde, Gunnar; Marinelli, Mattia; Træholt, Chresten

\section{Published in:}

Proceedings of $56<$ sup $>$ th $</$ sup $>$ International Universities Power Engineering Conference

Link to article, DOI:

10.1109/UPEC50034.2021.9548259

Publication date:

2021

Document Version

Peer reviewed version

Link back to DTU Orbit

Citation $(A P A)$ :

Pinter, Z. M., Papageorgiou, D., Rohde, G., Marinelli, M., \& Træholt, C. (2021). Repriew of Control Algorithms for Reconfigurable Battery Systems with an Industrial Example. In Proceedings of 56 International Universities Power Engineering Conference IEEE. https://doi.org/10.1109/UPEC50034.2021.9548259

\section{General rights}

Copyright and moral rights for the publications made accessible in the public portal are retained by the authors and/or other copyright owners and it is a condition of accessing publications that users recognise and abide by the legal requirements associated with these rights.

- Users may download and print one copy of any publication from the public portal for the purpose of private study or research.

- You may not further distribute the material or use it for any profit-making activity or commercial gain

- You may freely distribute the URL identifying the publication in the public portal 


\title{
Review of Control Algorithms for Reconfigurable Battery Systems with an Industrial Example
}

\author{
Zoltan Mark Pinter ${ }^{1}$, Dimitrios Papageorgiou ${ }^{1}$, Gunnar Rohde ${ }^{2}$, Mattia Marinelli ${ }^{1}$, and Chresten Træholt $^{1}$ \\ ${ }^{1}$ Department of Electrical Engineering, Technical University of Denmark, Kongens Lyngby, DK \\ ${ }^{2}$ Danish Center for Energy Storage, Copenhagen, DK \\ \{pinzo,dimpa\}@dtu.dk, gr@atv.dk, \{matm,ctr\}@dtu.dk
}

\begin{abstract}
Battery cells within battery energy storage systems (BESS) do not have homogeneous attributes, and the lowest capacity ones limit the performance and lifetime of the whole pack. Modern battery management systems (BMS) solve this problem with balancing, while providing the required service, and safe operation to the user. Reconfigurable battery systems (RBS) are BESSs that involve a BMS with reconfiguration. Reconfiguration uses feedback to determine the circuit switching logic. This paper presents a structured review of the control algorithms for RBSs. The RBSs are divided into groups according to their control strategies and control implementations. Finding the adequate control strategy requires well-defined objectives and control design. The control implementation focuses on physical and architectural aspects, like the reconfiguration frequency, the balancing operation and the control topology. The considerations and categories are discussed with the advantages, disadvantages and academic examples, and then an innovative industrial BMS is introduced.
\end{abstract}

Index Terms-Battery management systems, charge equalization, active balancing, reconfiguration, control strategy.

\section{INTRODUCTION}

An urgent topic of our decade is decarbonization and it is currently achieved by pouring large investments into renewable energy generation and electrical vehicles [1]. Fluctuations are imminent in the power generation and demand, therefore energy storage systems (ESS) become necessary. A promising commercial technology is the battery energy storage system, BESS. Furthermore, battery packs substitute fuel tanks in case of e-mobility.

Recently, lithium-ion battery cells are preferred in the industry due to their relatively high energy density and tolerance to large currents, but they are only durable in a restricted range of operating conditions [2]. This range is called the safe operating area (SOA). Lithium-ion cells are sensitive to overcharging or over-discharging, to extremes of voltages, currents, temperatures, and how often they are used.

Battery packs consist of a large number of battery cells. Due to manufacturing variations, the electrochemical properties of the cells are not homogeneous, resulting in different charge capacities, internal impedances, self-discharging rates and thermal properties [2]. For this reason, cell imbalance occurs,

This work has received funding from the EUDP and IFD funded TOPchargE project under the Grant Agreement No. 64019-0540 (http://topcharge.eu/) a term that refers to several physical properties differing for every cell.

The weakest cell always limits its neighbours because of its reduced operation range [3]. The current is limited due to increased internal impedance, and the capacity is limited as the weakest cell reaches its operation limit of state of charge (SoC) sooner. Predominantly, in a serial connection, the weakest cell limits the maximum current; in a parallel connection, the weakest cell limits the capacity. The varying internal resistance and the self-discharge increases the variance of SoCs of the cells along just some cycles. As the passing of cycles speeds up for the affected cells, it enhances aging. The internal resistances of the cells increase, limiting their performance. Furthermore, their capacity decreases. In other words, their state of health $(\mathrm{SoH})$ is reduced. In this indirect way, the weakest cell reduces the battery pack's lifetime as well.

\section{A. Battery management systems}

Battery management systems (BMS) are responsible for:

- fulfilling the service requirements from the user,

- protection of the cells, by limiting the usage to SOA,

- balancing the SoC of the cells to increase their performance and lifetime,

- thermal management,

- potentially fault tolerance.

The state-of-the-art BMS design techniques up to 2010 are described in [2]. These methods revolve mainly around estimation of SoC and $\mathrm{SoH}$, the balancing strategies (top balancing, midbalancing, bottom balancing), and the basic topologies made from commercial components. The differences between passive and active balancing are presented.

Balancing can be passive, when resistive elements convert the excess energy of higher SoC cells to heat. Balancing is active, when switching elements are used to redistribute the charge among the cells. Active battery equalization topologies are compared extensively in [4]. In this categorization, the topologies can be cell-bypass, cell-to-cell, cell-to-pack, packto-cell, or cell-to-pack-to-cell. Typically, to actively transfer charge between cells, an energy storage unit (ESU) (such as inductor, capacitor or transformer) is utilized. A counterexample is the pulse width modulation switching between parallelserial and serial-parallel circuit logics [5]. 
Active balancing methods may use fixed switching logic or reconfigurable switching logic. In case of fixed switching logic, cells may be connected occasionally in parallel. Alternatively, they can connect through an ESU, which helps to control the dynamics of the balancing current. Reconfigurable battery systems (RBS) utilize reconfigurable switching logic, using the estimation of the SoCs. They modify the switching logic according to a control strategy. This normally results in less unnecessary transfer of charge or lower rate of transfer. Hence copper losses are reduced compared to fixed switching logic, and variable voltage levels are possible at the point of connection of a battery string. On the other hand, requirements on circuit components are higher. Gates may experience more dynamic and larger range of voltages, and measurement units are expected to provide higher resolution to enable reliable feedback. The reviews like [6]-[10] summarize cell modeling, estimation of $\mathrm{SoC}$ and $\mathrm{SoH}$, control hardware and their topologies, fault accommodation, control design, scaling or granularity, and communication. Generally, the reconfiguration algorithms are strongly dependent on the circuit topology. In [10], it is discussed that there are still no strategies to design application specific RBSs, and with upscaling, or increasing algorithmic complexity, both computation time of optimization algorithms and the communication burden grow quickly. The most universal circuit topology is depicted in Fig. 1, which is capable of series, local parallel, global parallel connections and bypassing cells/modules. Other topologies can be provided by the simplification of it.

\section{B. Contribution}

The contribution of this paper pertains to analyzing control algorithms written for RBSs regarding both holistic and pragmatic perspectives. The potential design choices are listed with their benefits and drawbacks. Afterwards, the modular and commercial BMS, Nerve Smart System's NerveSwitch ${ }^{\circledR}$ [11] is shows an industrial aspect. It is compared to alternative solutions in the literature through categorizing the main traits of the reconfiguration control algorithms, while explaining the design choices.

NerveSwitch ${ }^{\circledR}$ is a BMS, which gains advantage in the market by eliminating the need of DC-DC converters, and by providing service and balancing the cells at the same time. Its modularity enables it to easily fit a large range of BESSs.

This paper is organized as follows. Section II reviews the control strategies for RBSs. Section III categorizes the literature according to physical and architectural considerations for implementing the control strategy. Section IV describes the novel commercial system, and motivates its categories with design choices. Section V discusses the outcomes of the paper.

\section{Control Strategy}

Lithium-ion systems provide high reliability in a safe operation area (SOA), which depends on SoC, current and temperature [2]. Hence at least a basic model of the cell and the topology is required for adequate usage. The models may rely on first principles from chemistry, thermodynamics or electricity (see equivalent circuit models, e.g. [12]) and empirical observations [13], [14]. Measurements, and these models are used to provide numerical values of how far the system is from its control objectives. The procedure for decision making follows a control design, which aims at meeting these targets.

Understanding the dominant dynamics of the cell helps to design an adequate control algorithm for the BESS. Using larger discharge current, the usable cell capacity decreases, which is the rate capacity effect. If sufficient relaxation time (scale of minutes) is given, the maximum open-circuit voltage belonging to the SoC is restored, this is the recovery effect. This dynamics and its dependency on temperature is described in [15]. Based on the temperature and the service power, optimal discharge currents and idle times can be selected for particular chemistries.

\section{A. Control Objectives}

The control objectives consist of safety measures, maintenance of the cells, providing the required service, and fault tolerance. With regards to the control system design, the time horizon of objectives is a paramount aspect. Objectives with identical time horizon may contradict each other and cause stability issues, furthermore, long term objectives are often neglected. The objectives can be constant, changing in a predefined manner, predictable or unpredictable. Accordingly, the control algorithm may use planning or prediction. For instance, the service power is predicted by a recursive least squares algorithm in [16].

Typically, the service related objectives are short term ones, like providing the required power, voltage, current or impedance, and maintaining the integrity of the BESS. Keeping the cells in the SOA of voltage, current and temperature ranges are prioritized as well. Additionally, fault accommodation may be considered to alleviate the effects of a failure [12], [17]. Faults can be short-circuit, open circuit, or thermal runaway [18]. Intermediate term objectives are the balancing of the SoCs, and utilizing maximal capacity by considering rate capacity and recovery effects. Note that balancing is sometimes regarded as an automatic consequence of the switching topologies used, hence it may be neglected from the objectives [19], [20]. The long term objective is to maximize the lifetime of the pack. This is the most challenging one, as the estimation of the main indicator of the aging, the $\mathrm{SoH}$, still divides the literature [2]. Aging depends on the combination of a lot of factors. Lifetime is prolonged by reducing the cell temperature in [21], but note that the internal resistance is then also larger [22].

\section{B. Control Design}

The control algorithm (Fig. 2) is the decision making process, which utilizes the model and measurements (or processed information from other control units, see Section III-C) to determine the state of the system. The algorithm determines the actuation based on comparing this state to the target state (or conveys it to other control units). The decision is 


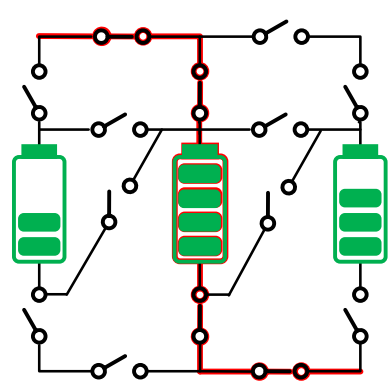

a)

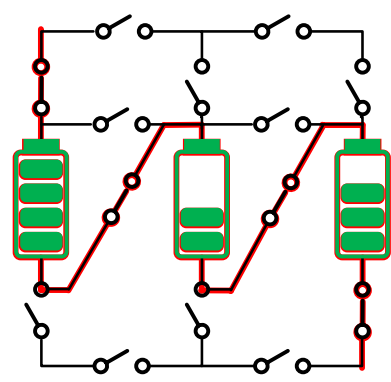

b)

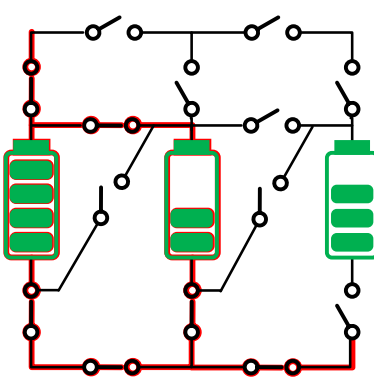

c)

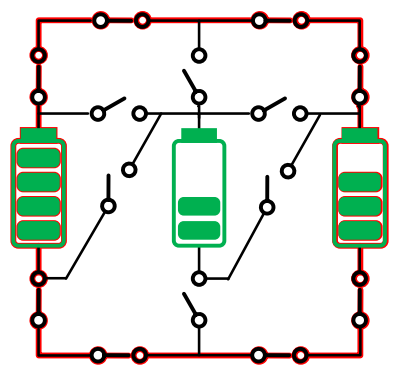

d)

Fig. 1. The most universal circuit topology with the possible switching logics: a) engaging the middle and bypassing the other cells; b) series connection; c) local parallel connection of the left cells; d) global parallel connection of the side cells

made about both the reconfiguration, and the allocated time durations of sublogics. The algorithm always involves a rule base, it is likely to use a model, and it may leverage some optimization, like data driven or graph-based control design as well. Note that the estimation of physical quantities covers a large independent area in the literature [23], hence it is not considered in this work.

Model based algorithms use either time domain or frequency domain (e.g. [24]) models. The emphasized dynamics is mostly linear, when considering the equivalent circuit model. Adding the dynamics of the chemistry, it becomes nonlinear [14], [15]. The distributed control units estimate SoC independently in [12], and their average is used to decide which modules to engage. The alignment is smoothed with low pass filters, and convergence of the estimations is proved.

Graph-based algorithms [3], [15] normally reconfigure the entire switching logic, which may lead to temporary pause of supply [19]. Generally speaking, these algorithms are finite state machines (FSM), as they have a finite number of states. They do path selection to address the problem. Unfortunately, the encountered path selection problems may be NP-hard [25]. Regarding data driven applications, like artificial neural networks or fuzzy systems [26], [27], there is not sufficient amount of user data yet today. A Markov model is described in [17] to overcome this problem. It is a typical example of a FSM, and it is straightforward to generate use cases with it.

For larger scale applications, the control design should be scalable [17]. In [28], the effect of the level and complexity of reconfiguration on the computation time is investigated, like reorganizing cells among modules. The genetic algorithm is stated as the ultimate control design solution for scale-wise unbounded systems, however, it is computationally expensive.

\section{CONTROL IMPLEMENTATION}

The control strategy aims at fulfilling control objectives, while using feedback for decision-making. Control implementation is the set of pragmatic design choices, with which the control strategy is fitted to the physical system. The implementation may consist of decisions about reconfiguration frequency, time allocation for balancing, or distributing control action.

\section{A. Reconfiguration frequency}

The reconfiguration switching logic can be

- static,

- dynamic, with static gate drive, and

- dynamic assisted with PWM.

Static reconfiguration updates the apparent circuit topology at the beginning or end of cycle intervals, like charging, discharging, balancing or idle modes [16], [29]. Dynamic reconfiguration updates more frequently, either being triggered by an event, or synchronized by the clock. Generally, static reconfiguration will have lower investment cost, while dynamic reconfiguration will provide better balancing performance and robustness to unknown factors. Some of the control strategies applied, especially with graph search, take longer computation time [28]. Graph search may also simplify the system with using FSMs [3], [17]. These factors limit the maximum reconfiguration frequency, and often motivate static reconfiguration.

Reconfiguration frequency influences the balancing performance and efficiency of RBSs [30]. Since reconfiguration is based on feedback, higher frequency increases the resolution requirements towards the measurement unit, with respect to both time and accuracy. Additionally, the requirement on reconfiguration delay is harsher. Selecting too high frequency may lead to circuit hazards. In [31], circuit hazards are avoided by temporary switching logic. Additional rule-base is considered to avoid pause in the service in [19]. Higher switching frequency increases switching and gate drive losses, but copper losses are reduced as well [30]. When ESUs are utilized [12], higher frequency eases the requirement on the size.

Pulse Width Modulation (PWM) is a widespread tool of control deployment [26], [32]. Optimal switching frequency is discussed with regards to capacity, efficiency and thermal characteristics in [33]. Meanwhile, high PWM frequencies decrease the lifetime of the battery [31].

\section{B. Balancing operation}

Balancing operation is challenging to define, since it can be either the intention of the designer, or an apparent topology ensuring the equalization of SoCs. It compensates for the variations caused by the difference of leakage currents, or 


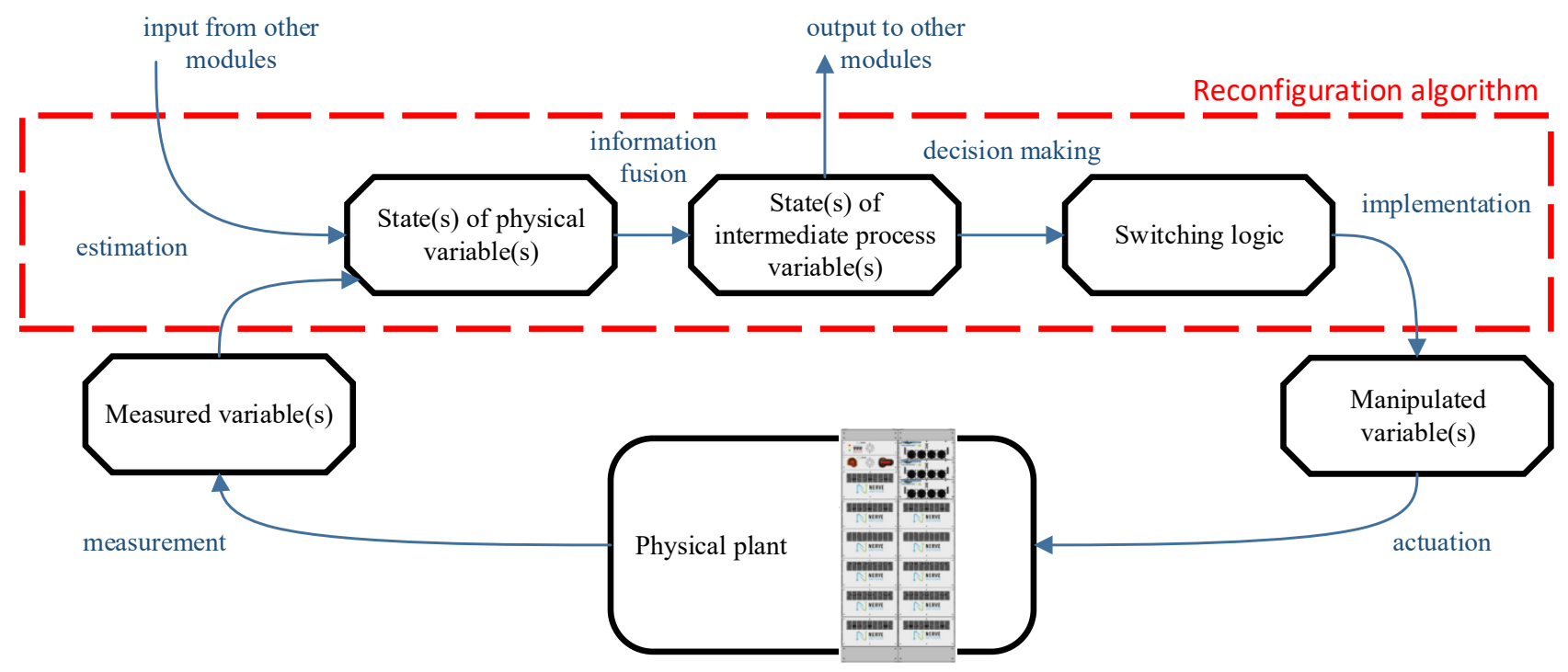

Fig. 2. The reconfiguration algorithm with the control loop

reduced capacities [2]. It can be done unintentionally as well, by occasionally connecting cells in parallel, like in [14].

The cell current could be categorized by its work. If the work is done along the electric field of a cell, it can be seen as service current. Otherwise, it is done between the anodes (or cathodes) of cells due to the difference of SoCs, and it can be seen as balancing current. However, dividing the service current among the cells also balances their $\mathrm{SoC}$ in an indirect way.

Regarding the control applications, they approach the duality of service and balancing by either providing dedicated time for balancing, or doing them simultaneously [8] (see Fig. 3). Hence balancing operation can be

- scheduled or

- integrated.

Generally speaking, scheduled balancing compromises service capability in time-usage, while integrated balancing compromises it in capacity-usage (since a fraction of the apparently used capacity does not carry out service). To provide the same power for the same circuit, scheduled balancing will need to use larger current, which faces rate capacity effect, and quicker aging due to larger temperatures [21]. Alternatively, integrated balancing uses less current, but there is less utilization of the recovery effect. Rate capacity effect of integrated balancing may be reduced by connecting cells with similar SoC in series with variable resistances [25]. However, this increases copper losses. In [16], the relations between utilizable capacity, discharge rates and operation/rest times are measured, and the two balancing operations are combined to achieve optimal performance.

\section{RBS control topology}

When a larger scale BESS is used, the distribution of control action has a fundamental effect on performance. The topology of the RBS control can be

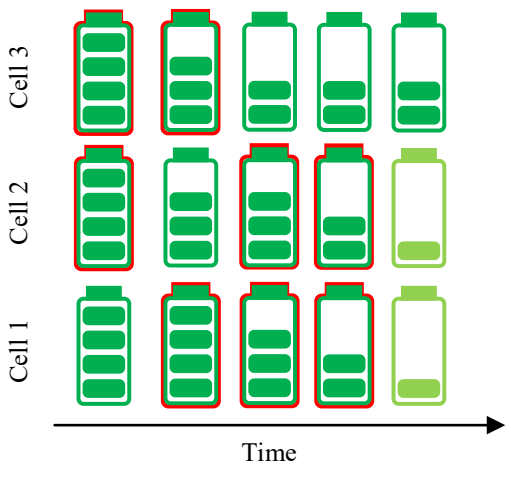

Fig. 3. Scheduled balancing. The two largest SoC cells are used each time instant, until the minimum SoC (light green) limits the operation

- centralized (flat),

- hierarchical (master-slave) or

- distributed,

(similarly to the BMS categories in [2]). Centralized RBS control makes control decisions in one central control unit. Typically, systems with complex switching topologies are better utilized with centralized RBS [3], [19], [28]. Hierarchical RBS control makes some of the control decisions locally for modules/cells, and these control units are ordered to fulfill commands from the central control unit. For example, feedback at lower level, like droop control can be managed by sending reference values from above [24]. A special example of hierarchical RBS control is the AC battery, where often a higher level control unit balances both on bank and cell level, while tracking the $\mathrm{AC}$ signal reference with a multilevel inverter [32]. In case of distributed RBS control, the control units work in a cooperative manner, without a central control unit. Typically for larger scales, they only cooperate with some neighbours. Centralized and hierarchical control 
systems are more robust to stability issues and are more likely to find global optimal operations than the distributed counterparts. On the other hand, they have higher computation burdens. Distributed and hierarchical RBS control has lower demands on their communication lines and are less prone to communication hazards than the centralized ones [12]. On the contrary, their operations are more prone to electromagnetic interference, since the control units are located closer to the high power electronics.

The modularity of a RBS control topology is the ability to attach additional modules/cells to the system or restructuring an already existing one without the necessity of re-configuring the control units. It is vital for commercial systems.

\section{The NerveSwitch®}

NerveSwitch ${ }^{\circledR}$ is a dynamic reconfiguration BMS, which provides reliable service without the need for DC-DC converters, while keeping the cells of the battery balanced. The control system is modular and hence applicable to different topologies and battery chemistries. Currently, its commercialised BESS uses 100 Ah lithium iron phosphate (LFP) cells, which provide solutions to concerns regarding cycle life, power density, charge-discharge current ranges, and there is no chance of thermal runaway [2]. A $312 \mathrm{kWh}$ pilot BESS powers a DC microgrid on the Danish island of Bornholm [34]. The simulated efficiencies are presented in [35], giving 93.3\% efficiency for EV fast charging at $50 \mathrm{~kW}$ charging level. Reconfiguration electronics cause $1.2 \%$ power loss.

The hierarchical RBS control has four levels (see Fig. 4). The control levels in a decreasing order of hierarchy are:

- Energy Management System (EMS),

- String Control Module (SCM),

- Module Controller (MC), and

- Cell Controller (CC).

The choice, which cells shall be engaged, is made by a complex rule-base constructed from professional expertise. The EMS focuses on the service perspective, considering the power, voltage, current and impedance requirements towards the battery pack. Furthermore, it also manages the equalization of the string SoCs. It can connect the battery strings in series or parallel through cross-over modules, which units determine the busbar topologies and communication connections.

The SCM focuses on the protection and maintenance of the battery cells, while also satisfies the service commands from the EMS. The SCM divides the cells into groups of engaged and bypassed ones. The number of engaged cells depends on the voltage requirement. The interface to the pack is either a DC link or an inverter, which is used at its optimal efficiency. In case of discharging, when the highest SoC cell of the engaged group reaches the highest SoC of the bypassed group, the cells are redistributed. This logic is to minimize the switching frequency, as the highest SoC cell of the bypassed group is likely to take the most time to become the highest SoC cell of the whole string. This way the recovery effect is maximized. At the same time, a group of cells having the lowest SoC is prioritized for engaging, for the whole discharge

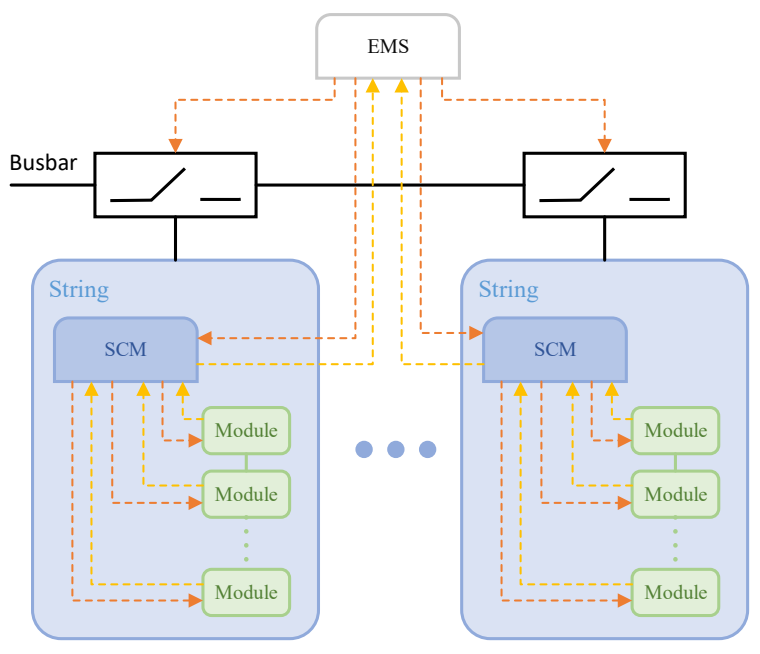

a)

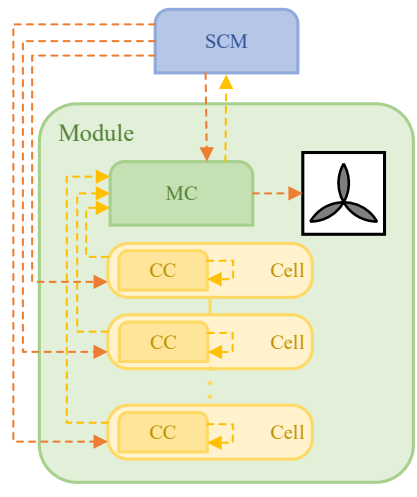

b)
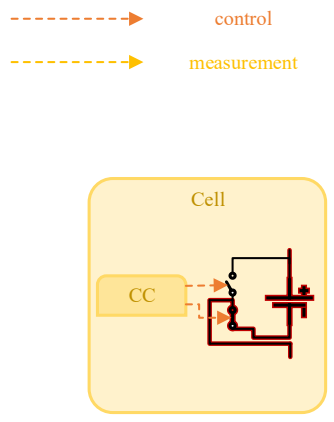

c)

Fig. 4. Levels of control: a) EMS and SCM, b) SCM, MC and CC c) CC

period. This is because the cells, which are further from $100 \%$ SoC, are the ones with more utilizable capacity. If they are prioritized, they get discharged first and get selected in the charging phase as well. Hence they are used more and the pack SoH is being balanced. Charging operation is analogous to the discharging one.

The MC does basic safety checks and controls the temperature within the module with a fan. The CC uses MOSFETs for either engaging or bypassing cells. It implements safety limits to keep the cell in the SOA, bypasses the cell in case of a fault, and it communicates its state towards the MC. The safety operations are directly triggered by the hazardous events.

\section{CONCLuSion}

This paper presents control algorithms for reconfiguration battery systems. It is structured to help the control design with motivations, drawbacks and corresponding examples from the literature. Reconfiguration algorithms use feedback to make decisions about actuation, and their use require both an abstract control design and pragmatic considerations. This is for the sake of the dynamical needs of the chemistries of 
the battery cells, reducing losses and avoiding circuit hazards. Finally, to broaden the perspective with a well-tried example, a commercial application is introduced.

There are further aspects of control algorithms, which could not be covered in the given volume. The estimation of physical variables of the system, like $\mathrm{SoC}$ and $\mathrm{SoH}$, is widely covered by the literature. Another aspect could be the physical level of reconfiguration; whether it is applied to cells or modules. It provides a trade-off between the investment cost and the effectiveness of the balancing. The effect of scaling on the selection of control design could be discussed as well.

As a result of the review, the following gaps are found in the literature. First, integrated balancing is still not implemented sufficiently, most probably, as it requires more sophisticated design. With regards to control algorithms, predictive control design is not discovered yet. Moreover, since there is not much available data of use cases, use case generation could be enhanced to advance data driven designs. Then learning, for instance reinforcement learning, may be the solution to further improve RBS control.

\section{REFERENCES}

[1] L. Meng, Q. Shafiee, G. F. Trecate, H. Karimi, D. Fulwani, X. Lu, and J. M. Guerrero, "Review on control of dc microgrids and multiple microgrid clusters," IEEE Journal of Emerging and Selected Topics in Power Electronics, vol. 5, no. 3, pp. 928-948, 2017.

[2] D. Andrea, Battery management systems for large lithium-ion battery packs. Artech house, 2010.

[3] L. He, Z. Yang, Y. Gu, C. Liu, T. He, and K. G. Shin, "Soh-aware reconfiguration in battery packs," IEEE Transactions on Smart Grid, vol. 9, no. 4, pp. 3727-3735, 2016.

[4] J. Gallardo-Lozano, E. Romero-Cadaval, M. I. Milanes-Montero, and M. A. Guerrero-Martinez, "Battery equalization active methods," Journal of Power Sources, vol. 246, pp. 934-949, 2014.

[5] X. Wang, B. Duan, Y. Shang, Y. Xu, K. Yu, and C. Zhang, "Fast equalization for lithium ion battery packs based on reconfigurable battery structure," in 2020 IEEE/IAS Industrial and Commercial Power System Asia (I\&CPS Asia). IEEE, 2020, pp. 1149-1154.

[6] V. Viswanathan, L. N. Palaniswamy, and P. B. Leelavinodhan, "Optimization techniques of battery packs using re-configurability: A review," Journal of Energy Storage, vol. 23, pp. 404-415, 2019.

[7] $\mathrm{S} . \mathrm{Ci}, \mathrm{N}$. Lin, and D. Wu, "Reconfigurable battery techniques and systems: A survey," IEEE Access, vol. 4, pp. 1175-1189, 2016.

[8] S. Muhammad, M. U. Rafique, S. Li, Z. Shao, Q. Wang, and X. Liu, "Reconfigurable battery systems: a survey on hardware architecture and research challenges," ACM Transactions on Design Automation of Electronic Systems (TODAES), vol. 24, no. 2, pp. 1-27, 2019.

[9] J. Xu, Y. Li, C. Peng, X. Mei, and J. Wang, "A review on the battery balancing and reconfiguration methods," DEStech Transactions on Environment, Energy and Earth Sciences, no. iceee, 2018.

[10] W. Han, T. Wik, A. Kersten, G. Dong, and C. Zou, "Next-generation battery management systems: dynamic reconfiguration," IEEE Industrial Electronics Magazine, vol. 14, no. 4, pp. 20-31, 2020.

[11] "Home," Jan 2021. [Online]. Available: https://nervesmartsystems.com/

[12] T. Morstyn, M. Momayyezan, B. Hredzak, and V. G. Agelidis, "Distributed control for state-of-charge balancing between the modules of a reconfigurable battery energy storage system," IEEE Transactions on Power Electronics, vol. 31, no. 11, pp. 7986-7995, 2015.

[13] L. Calearo, A. Thingvad, and M. Marinelli, "Modeling of battery electric vehicles for degradation studies," in 2019 54th International Universities Power Engineering Conference (UPEC). IEEE, 2019, pp. 1-6.

[14] T. Kim, W. Qiao, and L. Qu, "Power electronics-enabled self-x multicell batteries: A design toward smart batteries," IEEE Transactions on Power Electronics, vol. 27, no. 11, pp. 4723-4733, 2012.

[15] N. Lin, S. Ci, D. Wu, and H. Guo, "An optimization framework for dynamically reconfigurable battery systems," IEEE Transactions on Energy Conversion, vol. 33, no. 4, pp. 1669-1676, 2018.
[16] H. Kim and K. G. Shin, "Scheduling of battery charge, discharge, and rest," in 2009 30th IEEE Real-Time Systems Symposium. IEEE, 2009, pp. $13-22$.

[17] _ , "Desa: Dependable, efficient, scalable architecture for management of large-scale batteries," IEEE Transactions on Industrial Informatics, vol. 8, no. 2, pp. 406-417, 2011.

[18] _ _ "On dynamic reconfiguration of a large-scale battery system," in 2009 15th IEEE Real-Time and Embedded Technology and Applications Symposium. IEEE, 2009, pp. 87-96.

[19] L. He, L. Gu, L. Kong, Y. Gu, C. Liu, and T. He, "Exploring adaptive reconfiguration to optimize energy efficiency in large-scale battery systems," in 2013 IEEE 34th Real-Time Systems Symposium. IEEE, 2013, pp. 118-127.

[20] F. Jin and K. G. Shin, "Pack sizing and reconfiguration for management of large-scale batteries," in 2012 IEEE/ACM Third International Conference on Cyber-Physical Systems. IEEE, 2012, pp. 138-147.

[21] R. L. Velho, J. Pombo, J. Fermeiro, M. Calado, and S. Mariano, "Lookup table based intelligent charging and balancing algorithm for li-ion battery packs," in 2018 International Conference on Intelligent Systems (IS). IEEE, 2018, pp. 209-214.

[22] M. Thingvad, L. Calearo, A. Thingvad, R. Viskinde, and M. Marinelli, "Characterization of nme lithium-ion battery degradation for improved online state estimation," in 2020 55th International Universities Power Engineering Conference (UPEC). IEEE, 2020, pp. 1-6.

[23] D. N. How, M. Hannan, M. H. Lipu, and P. J. Ker, "State of charge estimation for lithium-ion batteries using model-based and data-driven methods: A review," IEEE Access, vol. 7, pp. 136116-136 136, 2019.

[24] M. M. U. Rehman, F. Zhang, R. Zane, and D. Maksimovic, "Control of bidirectional $\mathrm{dc} / \mathrm{dc}$ converters in reconfigurable, modular battery systems," in 2017 IEEE Applied Power Electronics Conference and Exposition (APEC). IEEE, 2017, pp. 1277-1283.

[25] L. He, L. Kong, S. Lin, S. Ying, Y. Gu, T. He, and C. Liu, "Reconfiguration-assisted charging in large-scale lithium-ion battery systems," in 2014 ACM/IEEE International Conference on CyberPhysical Systems (ICCPS). IEEE, 2014, pp. 60-71.

[26] T. T. N. Nguyen, H.-G. Yoo, S. K. Oruganti, and F. Bien, "Neuro-fuzzy controller for battery equalisation in serially connected lithium battery pack," IET power electronics, vol. 8, no. 3, pp. 458-466, 2015.

[27] X. Cui, W. Shen, Y. Zhang, and C. Hu, "Fuzzy logic controller for battery balancing system for lithium-iron phosphate battery pack," in 2017 12th IEEE Conference on Industrial Electronics and Applications (ICIEA). IEEE, 2017, pp. 1723-1728.

[28] W. Han, C. Zou, L. Zhang, Q. Ouyang, and T. Wik, "Near-fastest battery balancing by cell/module reconfiguration," IEEE Transactions on Smart Grid, vol. 10, no. 6, pp. 6954-6964, 2019.

[29] K.-M. Lee, D.-H. Kim, J.-H. Shin, T.-D. Goh, J.-H. Park, and Y.-H Ryu, "Reconfigurable battery system with active balancing circuit," in IECON 2019-45th Annual Conference of the IEEE Industrial Electronics Society, vol. 1. IEEE, 2019, pp. 4583-4586.

[30] Z. Zhang, Y.-Y. Cai, Y. Zhang, D.-J. Gu, and Y.-F. Liu, "A distributed architecture based on microbank modules with self-reconfiguration control to improve the energy efficiency in the battery energy storage system," IEEE Transactions on Power Electronics, vol. 31, no. 1, pp. 304-317, 2015.

[31] G. Wang, J. Pou, and V. G. Agelidis, "Reconfigurable battery energy storage system for utility-scale applications," in IECON 2015-41st Annual Conference of the IEEE Industrial Electronics Society. IEEE, 2015, pp. 004 086-004091.

[32] E. Chatzinikolaou and D. J. Rogers, "Hierarchical distributed balancing control for large-scale reconfigurable ac battery packs," IEEE Transactions on Power Electronics, vol. 33, no. 7, pp. 5592-5602, 2017.

[33] F. Helling, J. Glueck, A. Singer, H.-J. Pfisterer, and T. Weyh, "The ac battery-a novel approach for integrating batteries into ac systems," International Journal of Electrical Power \& Energy Systems, vol. 104, pp. 150-158, 2019.

[34] T. Gabderakhmanova, J. Engelhardt, J. M. Zepter, T. M. Sørensen, K. Boesgaard, H. H. Ipsen, and M. Marinelli, "Demonstrations of dc microgrid and virtual power plant technologies on the danish island of bornholm," in 2020 55th International Universities Power Engineering Conference (UPEC). IEEE, 2020, pp. 1-6.

[35] J. Engelhardt, J. M. Zepter, T. Gabderakhmanova, G. Rohde, and M. Marinelli, "Double-string battery system with reconfigurable cell topology operated as a fast charging station for electric vehicles," Energies, vol. 14, no. 9, p. 2414, 2021. 